\title{
The auditory pathways: Startle amplitude and fear in an acoustic startle response paradigm in rats
}

\author{
ROBERT N. LEATON and JEAN M. KELSO \\ Dartmouth College, Hanover, New Hampshire
}

\begin{abstract}
Startle amplitude and startle-stimulus-induced freezing were measured in a standard acoustic startle response (ASR) paradigm in rats. Subsequently conditioned freezing was measured to an auditory conditioned stimulus (CS) with a foot-shock unconditioned stimulus (US). In Experiment 1, lesions to the brachium of the inferior colliculus (BIC) increased ASR amplitude and freezing. Lesions to the nucleus of the trapezoid body abolished ASR and marginally reduced freezing. Neither lesioned group differed from controls in conditioned freezing to the auditory CS. In Experiment 2, lesions to the medial geniculate nucleus (MGN) increased ASR amplitude, as did BIC lesions, but reduced freezing, while BIC lesions increased freezing. MGN lesions, unlike BIC lesions, significantly attenuated freezing to the auditory CS, as compared with controls. Both groups startled less than controls to a weak shock stimulus. The data suggest an ascending auditory pathway medial to BIC that carries the fear-inducing dimension of an acoustic stimulus and a descending pathway in BIC that provides tonic inhibition of the acoustic input to the ASR circuitry.
\end{abstract}

Several experiments suggest that Pavlovian fear conditioning occurs during the course of habituation training in a standard acoustic startle response (ASR) paradigm (Borszcz, Cranney, \& Leaton, 1989; Leaton \& Cranney, 1990; Young, Helmstetter, Rabchenuk, \& Leaton, 1991; Young \& Leaton, 1994). The contextual cues of the startle apparatus serve as the conditioned stimulus (CS), and the startle stimulus itself serves as the unconditioned stimulus (US). From this perspective, startle stimuli have two primary effects: They induce the short-latency startle response, and they serve as a US for fear conditioning. This conditioning can be indexed by freezing and associated heart-rate changes that develop in the startle chamber over the course of habituation training (Borszcz et al., 1989; Leaton \& Cranney, 1990; Young \& Leaton, 1994). The conditioned fear response elevates startle amplitudes on subsequent trials and may attenuate apparent habituation. In the ASR paradigm, lesions of the amygdala attenuate both the freezing and the heart rate changes associated with presentation of startle stimuli (Leaton \& Supple, 1987; Young \& Leaton, 1996).

The role of the amygdala in conditioned fear in the ASR paradigm is consistent with many reports of the role

This research was supported by the Department of Psychological and Brain Sciences and the Office of the Dean of the Faculty, Dartmouth College. Portions of Experiment 1 were presented at the 66th Annual Meeting of the Eastern Psychological Association, Boston, 1995. Portions of Experiment 2 were presented at the 68th Annual Meeting of the Eastern Psychological Association, Boston, 1998. Experiment 2 was submitted by J.M.K. as part of the requirements for the master's degree in psychology at Dartmouth College. Thanks are due Wesley P. Jordan for comments on an earlier version of this manuscript. Correspondence should be addressed to R. N. Leaton, Department of Psychological and Brain Sciences, 6207 Moore Hall, Dartmouth College, Hanover, NH 03755 (e-mail: leaton@dartmouth.edu). of the amygdala in more traditional fear conditioning paradigms. Amygdala lesions block fear-potentiated startle (Hitchcock \& Davis, 1986), reduce freezing to either an experimental context or a discrete CS associated with shock (Helmstetter, 1992; Phillips \& LeDoux, 1992), impair passive avoidance performance (Grossman, Grossman, \& Walsh, 1975), and block fear-conditioned heart rate changes (Kapp, Frysinger, Gallagher, \& Haselton, 1979; for recent reviews of this literature, see Davis, 1992, and LeDoux, 1995).

In a series of experiments, LeDoux and his colleagues (Iwata, LeDoux, Meeley, Arneric, \& Reis, 1986; LeDoux, Ruggiero \& Reis, 1985; LeDoux, Sakaguchi, \& Reis, 1984; Romanski \& LeDoux, 1992) have shown that the medial geniculate nucleus (MGN), the primary thalamic auditory relay nucleus, projects directly to the amygdala in addition to its known projections to the auditory cortex. The auditory cortex, in turn, projects to the amygdala, providing the amygdala with two sources of auditory information. Either of these pathways alone appears to be sufficient to support fear conditioning using an auditory CS paired with a foot-shock US. Lesions to the MGN that are large enough to interrupt both of these pathways block fear conditioning to an auditory CS.

Does the acoustic stimulus as US for fear conditioning in the ASR paradigm reach the amygdala by the same neural pathway as does the acoustic stimulus as CS? There is some evidence that the MGN may be critical in processing auditory USs. Bellgowan and Helmstetter (1996) have shown that hypoalgesia can be induced by acute exposure to a white-noise stimulus. This hypoalgesia is blocked by large electrolytic lesions to the MGN, as well as by lesions to the amygdala. These findings suggest that the MGN is critical in processing an auditory stimulus as US, as well as CS. However, in a preliminary experiment 
(Leaton \& Kelso, 1994), we found that electrolytic lesions of the MGN, contrary to our expectations, did not block freezing in an acoustic startle paradigm. Indeed, the lesioned animals spent more time freezing in the startle context than did sham-operated controls, and they also showed significantly elevated startle amplitudes. The present experiments were planned to further investigate (1) the role of the MGN in carrying the fear-provoking dimensions of an acoustic stimulus to the amygdala and (2) the role of the auditory pathways in modulating the amplitude of the startle response.

\section{EXPERIMENT 1}

The brachium of the inferior colliculus (BIC) provides the primary ascending auditory input from the inferior colliculus (IC) to the MGN (Winer, 1991). Bilateral lesions of BIC should significantly reduce auditory input to MGN and thereby limit auditory input to the amygdala. Such lesions should attenuate the effectiveness of an auditory stimulus as either CS or US for fear conditioning. Experiment 1 measured fear, as indexed by freezing behavior, in rats in a standard ASR paradigm following bilateral lesions of BIC. A sham-operated group and a group with bilateral lesions of the nucleus of the trapezoid body (TZ) were used for comparison. The TZ lesions were expected to block the startle response by blocking auditory input to the startle circuit and thus allow for the assessment of freezing in an animal with a much reduced response. Following the ASR testing, animals were tested in a shock-conditioning paradigm with an auditory CS to partially assess the situational generality of the results.

\section{Method}

\section{Subjects}

Thirty male Long-Evans-derived rats born and raised in the Dartmouth College Psychology Department vivarium were 110-120 days old at the time of surgery. They were maintained on a 14:10-h light:dark cycle (lights on at 0600) and tested between 1000 and $1600 \mathrm{~h}$. They were individually housed and had ad-lib access to food and water. They were divided into three groups matched on weight (mean weight $=426 \mathrm{~g}$ ). One group (BIC) received bilateral lesions of the brachium of the inferior colliculus $(n=11)$, one group (TZ) received bilateral lesions of the nucleus of the trapezoid body $(n=9)$, and one group (sham) received sham operations $(n=10)$.

\section{Surgery and Histology}

All surgery was performed aseptically under ketamine anesthesia ( $100 \mathrm{mg} / \mathrm{kg}$ ) supplemented with xylazine $(5 \mathrm{mg} / \mathrm{kg})$. All animal protocols were approved by the Institute Animal Care and Use Committee. The rats were mounted in a stereotaxic frame with ear bars, and care was taken to avoid damage to the ear drums. For all lesions, the initial coordinates were taken from the atlas of Paxinos and Watson (1986) and then adjusted from the histology of a series of preliminary animals. Coordinates were referenced to the interaural line (IAL) with the skull flat. The BIC was interrupted bilaterally by lowering a stainless steel razor blade sliver $(1.0 \mathrm{~mm}$ wide) through the fiber bundle under stereotaxic control. The coordinates were $2.0 \mathrm{~mm}$ anterior and $3.0 \mathrm{~mm}$ dorsal to IAL. The medial part of the blade was lowered $2.2 \mathrm{~mm}$ lateral to the midline and then moved $0.6 \mathrm{~mm}$ lateral and removed from the brain. The TZ lesions were made by passing anodal current $(1.4 \mathrm{~mA}$ for $10 \mathrm{sec})$ through a stereotaxically oriented stainless steel insect pin insulated except for $0.5 \mathrm{~mm}$ of the tip. The coordinates were $0.3 \mathrm{~mm}$ anterior to and $0.4 \mathrm{~mm}$ below IAL and $1.1 \mathrm{~mm}$ lateral to the midline. The shamoperated rats were anesthetized and mounted in the stereotaxic frame. Their skulls were bared, but no holes were drilled.

At the conclusion of testing, lesioned rats were administered a lethal dose of sodium pentobarbital $(100 \mathrm{mg} / \mathrm{kg})$ and perfused intracardially with normal saline followed by $10 \%$ buffered formalin. The brains were removed from the skulls and stored in $10 \%$ buffered formalin for $24 \mathrm{~h}$ and then transferred to a $20 \%$ sucroseformalin solution for a further $24 \mathrm{~h}$. Coronal sections were cut at $40 \mu \mathrm{m}$ on a freezing microtome. Every third section was mounted and stained with cresyl violet. The extent of the lesion was assessed by reconstructing the lesions on diagrams derived from the Paxinos and Watson (1986) brain atlas.

\section{Apparatus}

Acoustic startle. The startle apparatus was a modification of one described in detail previously (Leaton, 1976). The rats were tested in one of two $20 \times 12 \times 14 \mathrm{~cm}$ holding cages, fabricated from aluminum strips, except for one Plexiglas end wall and a floor consisting of $2.5-\mathrm{mm}$ brass rods fixed to Phenolite insulating material. Each cage was sandwiched between compression springs attached to a rigid superstructure, housed within a separate sound attenuating chamber, and illuminated by a $7-\mathrm{W}$ white incandescent bulb. Vertical displacement of the chamber moved an attached magnet within a fixed coil, inducing a voltage that was amplified, dig. itized $(10 \mathrm{kHz})$, rectified, and integrated by a microcomputer system. Startle amplitude was measured as the integrated voltage of the 200-msec epoch beginning at the onset of the startle stimulus A window in the sound-attenuating chamber allowed a clear view of the rat from the darkened test room.

The startle stimulus was a $117-\mathrm{dB}, 100-\mathrm{msec}$ white-noise burst with $0.4-\mathrm{msec}$ rise/fall time delivered by a $9-\mathrm{cm}$ piezoelectric tweeter (Herald Electronics) centered $12 \mathrm{~cm}$ from the long wall of the cage. Background white noise of 66-dB SPL was delivered continuously by a $10-\mathrm{cm}$ speaker mounted above the tweeter. Auditory intensities were measured with a Quest impulse sound-level meter (Model 2700, A scale, max) with the microphone centered inside the startle chamber.

Shock conditioning. Shock training took place within two identical $23.5 \times 29 \times 19.5 \mathrm{~cm}$ observation chambers. The end walls were stainless steel, and the other two were clear Plexiglas. The grid floor was composed of $2.5-\mathrm{mm}$ stainless steel rods spaced $1.25 \mathrm{~cm}$ apart. Scrambled shock $(1 \mathrm{~mA}, .5 \mathrm{sec})$ was delivered to the grid floor by a Grayson-Stadler (Model 700) shock generator. Each chamber was housed within one of the sound-attenuating chambers described above and illuminated by a $7-\mathrm{W}$ white incandescent bulb. Two wire mesh cylinders were used to provide for testing in a changed context. The cylinders $33 \mathrm{~cm}$ in diameter and $30.5 \mathrm{~cm}$ high) had a solid wooden floor and an open top. The top wooden support provided a $2.5-\mathrm{cm}$ lip around the upper circumference of the cylinder. Each cylinder was housed within one of the same sound-attenuating chambers described above, and the roof of the chamber provided the top of the cylinder. Illumination was provided by the 7-W white incandescent bulb.

The CS was an 80-dB (SPL, A-scale, slow), $3-\mathrm{kHz}$ pure tone with a $20-\mathrm{sec}$ duration and an $80-\mathrm{msec}$ rise/fall time. The CS was superimposed on $70-\mathrm{dB}$ background white noise.

\section{Procedure}

Acoustic startle. Testing began a minimum of 14 days after surgery. Following three daily 5 -min preexposure sessions in the startle chambers, all rats received eight presentations of the $117-\mathrm{dB}$, 100 -msec white-noise startle stimulus on a 40 -sec interstimulus interval in each of five sessions given on consecutive days. The first 

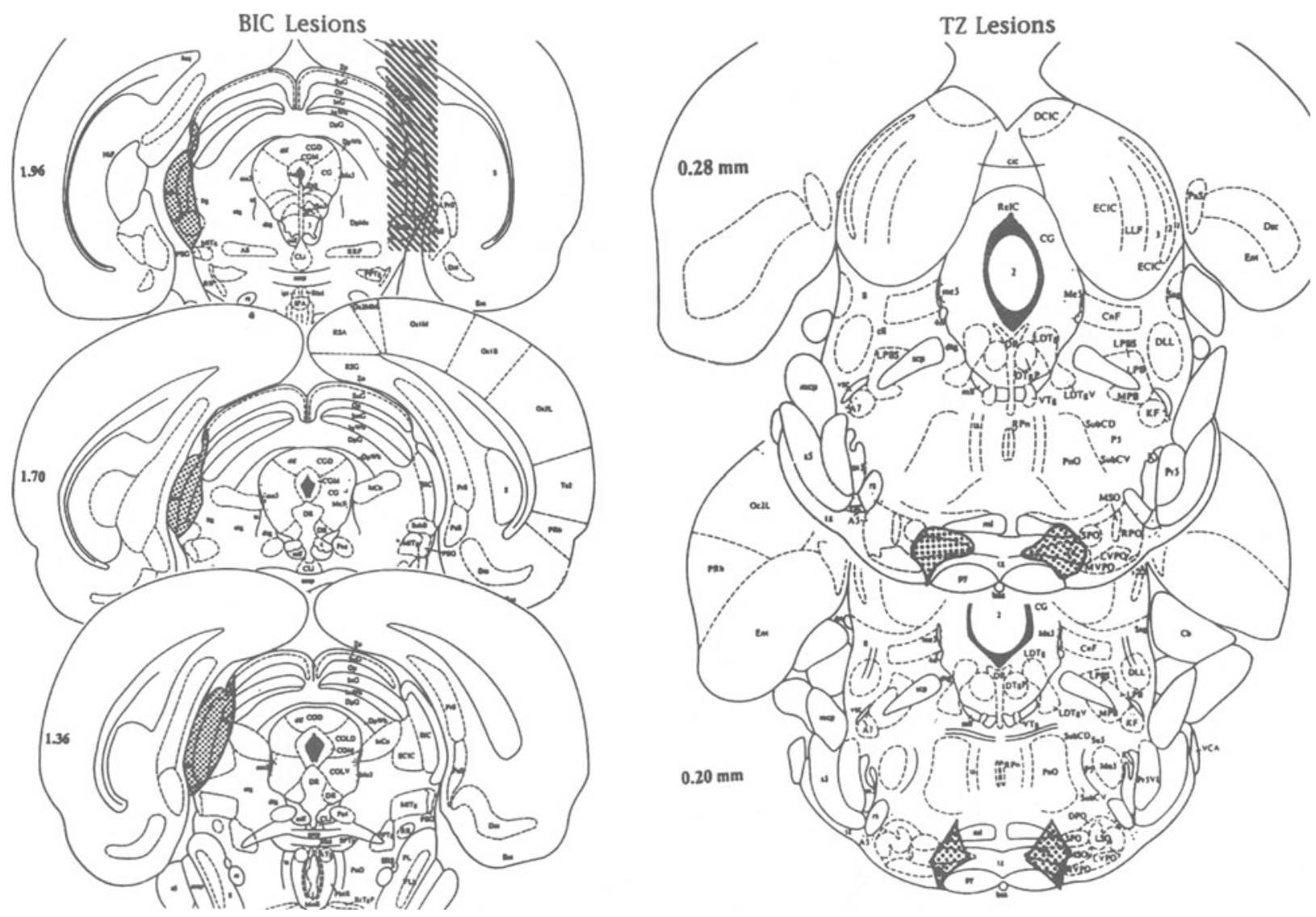

Figure 1. Diagrammatic reconstructions of representative lesions in Experiment 1 . The left side of the figure shows a representative lesion to the brachium of the inferior colliculus (BIC). The lesions were closely bilaterally symmetrical. The top figure on the right side of the BIC diagrams shows the intended track of the cutting blade. The right side of the figure shows a representative lesion to the nucleus of the trapezoid body (TZ). The numbers at the left of the diagrams are millimeters anterior to the interaural line. Adapted from The Rat Brain in Stereotaxic Coordinates (2nd ed., Figures $46-48$ and 52-53), by G. Paxinos and C. Watson, 1986, New York: Academic Press. Copyright 1986 by Academic Press. Adapted with permission.

stimulus in each session was presented $90 \mathrm{sec}$ into the session, and the rats were removed from the startle chambers $60 \mathrm{sec}$ following the last stimulus. Freezing was measured using an observational time-sampling procedure. The rats were observed every $2 \mathrm{sec}$ for the $20 \mathrm{sec}$ prior to each startle stimulus. Their behavior was judged at each of these 10 observation points as freezing or not freezing. Freezing was defined as the absence of all movement, including nose and whiskers but excluding the movements necessitated by breathing (Fanselow, 1984). These observations were converted to a trial-by-trial percentage freezing measure. Observers were blind to group identity.

Shock conditioning. Shock training began 2 weeks following the last startle test. On consecutive days, each rat was given (1) a 5min preexposure session in the shock chamber, (2) two daily sessions with two presentations of the 20 -sec CS alone, and (3) five daily training sessions with two CS-shock pairings each session. On CS-alone days and training days, the first trial was presented 120-180 sec after the session began, and the second trial occurred $120-180 \mathrm{sec}$ later. The rats were removed $1 \mathrm{~min}$ following the last trial. On training trials, the shock was presented at CS offset. On all of these trials, freezing was measured, as described above, during the $20-\mathrm{sec}$ period preceding the CS (the preCS period) and during the 20 -sec CS period. One day after the fifth training session, the rats were tested in the changed context. On consecutive days, they received (1) one preexposure session to the new context with no $\mathrm{CS}$ presentations but with freezing measured when the CS would have been presented and (2) two daily sessions with presentation of the CS alone. The CS was presented on the same time schedule as in training, and freezing was measured as before. Observers were blind to group identity.

Statistical analysis. Most statistical analyses were carried out using a mixed between-within analysis of variance (ANOVA). Single degree of freedom polynomial contrasts were used to determine the effects of within-subjects factors. Unless otherwise stated, $F$ statistics and significance levels are given only for first-order polynomials.

\section{Results}

\section{Histology}

Representative lesions are shown diagrammatically in Figure 1. The left side of the figure shows typical damage for a BIC lesion on the left of the brain sections; the right side of the top section shows the intended tract of the cutting blade. The lesions closely approximated bilateral symmetry, and the BIC was completely interrupted in all animals. In addition to the BIC damage, the nucleus of the 
A. Startle: Day 1

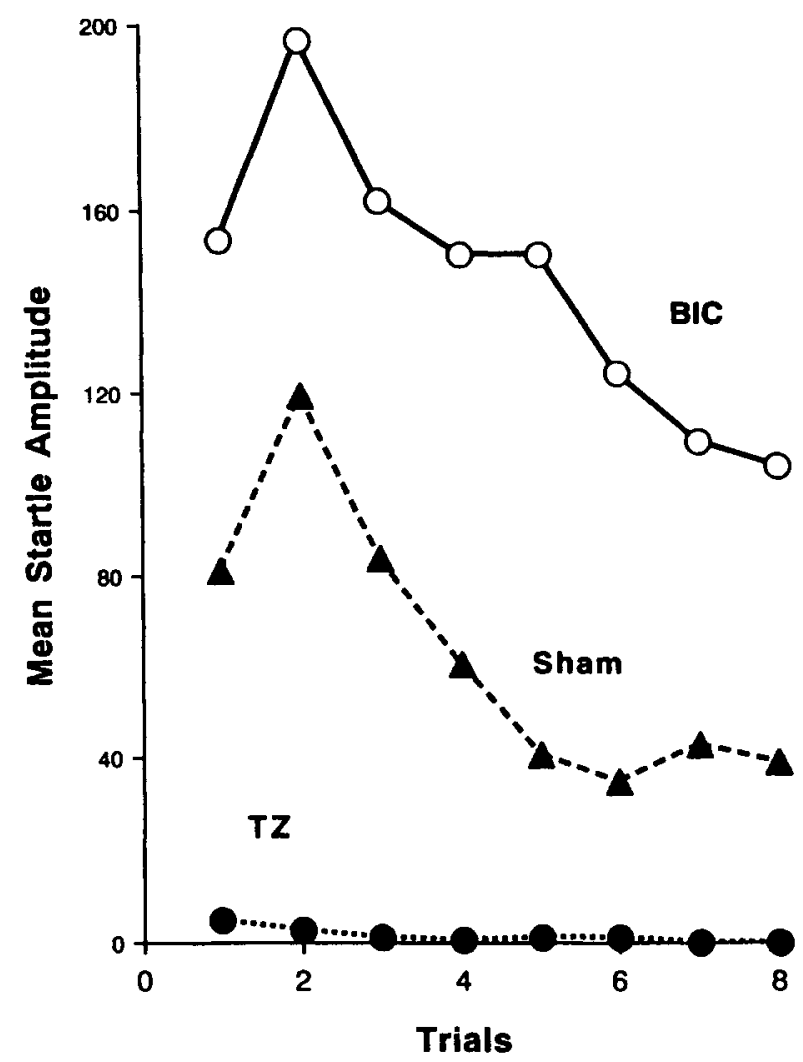

B. Freezing: Day 1

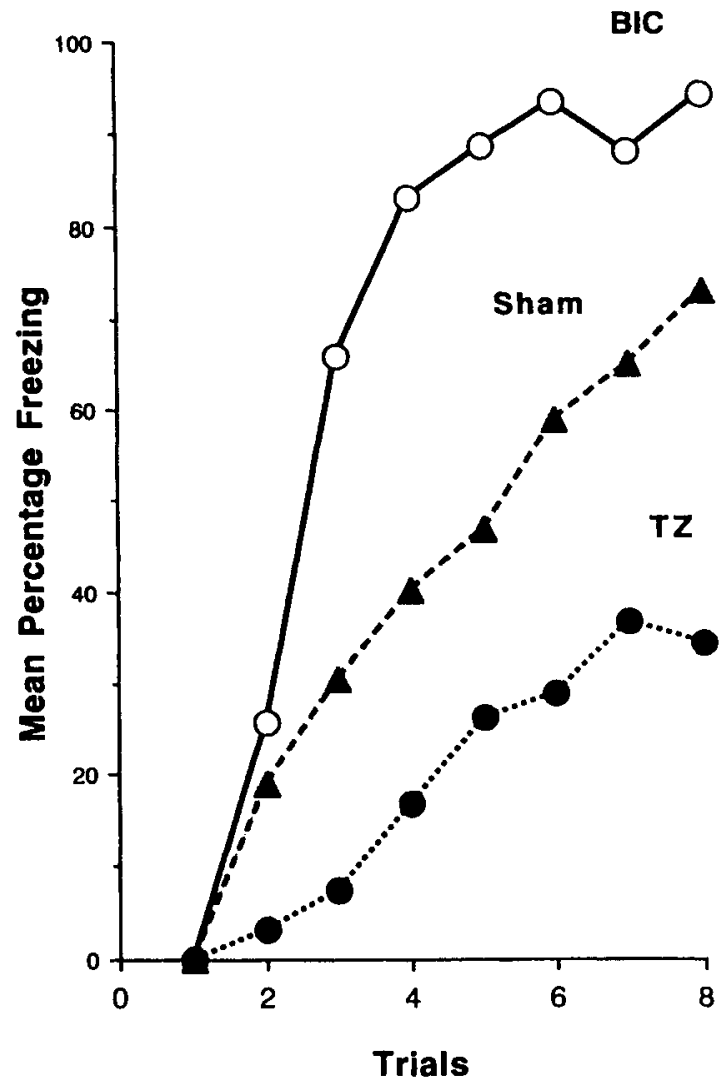

Figure 2. (A) Mean startle amplitude for the three groups over the eight trials on the first acoustic startle response test day in Experiment 1. (B) Mean percentage freezing for the three groups on the first acoustic startle response test day in Experiment 1. Freezing was measured for the 20 sec preceding each of the eight startle stimuli. The BIC group had lesions to the brachium of the inferior colliculus, the $T Z$ group had lesions to the nucleus of the trapezoid body, and the sham group received sham operations.

BIC was consistently damaged bilaterally, as was the subbrachial nucleus and the lateral edges of the superior colliculus. The external cortex of the inferior colliculus was damaged in some cases, but this damage was slight and not consistently bilateral. The overlying cortex received some damage from the tract of the blade.

The right side of the figure shows a representative lesion to TZ. The TZ was almost completely destroyed bilaterally in all animals. The damage to surrounding structures was limited and inconsistent. The lesions invariably broke through the ventral surface of the brain, and the tract of the $T Z$ was completely severed bilaterally.

\section{Startle Testing}

Startle. The ASR for the first test day is shown in Figure 2A. The BIC group showed the highest startle amplitudes, whereas the $T Z$ group showed little or no startle response. An ANOVA over the eight trials yielded a significant group difference $[F(2,27)=21.56, p<.001]$, a significant trials effect $[F(1,27)=32.90, p<.001]$, and a significant groups $\times$ trials interaction $[F(2,27)=6.34$, $p<.01]$. Post hoc analysis (Tukey) of the group means showed that the BIC group responded at a significantly higher level than both the sham group $(p<.01)$ and the TZ group $(p<.001)$, and the sham group responded at a significantly higher level than the TZ group $(p<.05)$. This pattern of differences held over the 5 test days, but the differences among the groups diminished over days. The overall group difference was still significant on Day 5 $[F(2,27)=7.21, p<.01]$, but the difference between the BIC and sham groups, while still apparent, was no longer statistically significant $(p>.25)$.

Freezing. The freezing data for the first ASR test day are shown in Figure 2B. The BIC group showed a higher percentage of freezing than the sham group, which in turn froze more than the TZ group. All groups increased freezing over trials as they experienced the startle stimuli. An ANOVA over the eight trials yielded a significant group difference $[F(2,27)=12.66, p<.001]$, a significant trials effect $[F(1,27)=82.56, p<.001]$, and a significant groups $\times$ trials interaction $[F(2,27)=3.72, p<$ $.05]$. Post hoc analysis of the group means showed that 


\section{A. Training: CS on Trial 1}

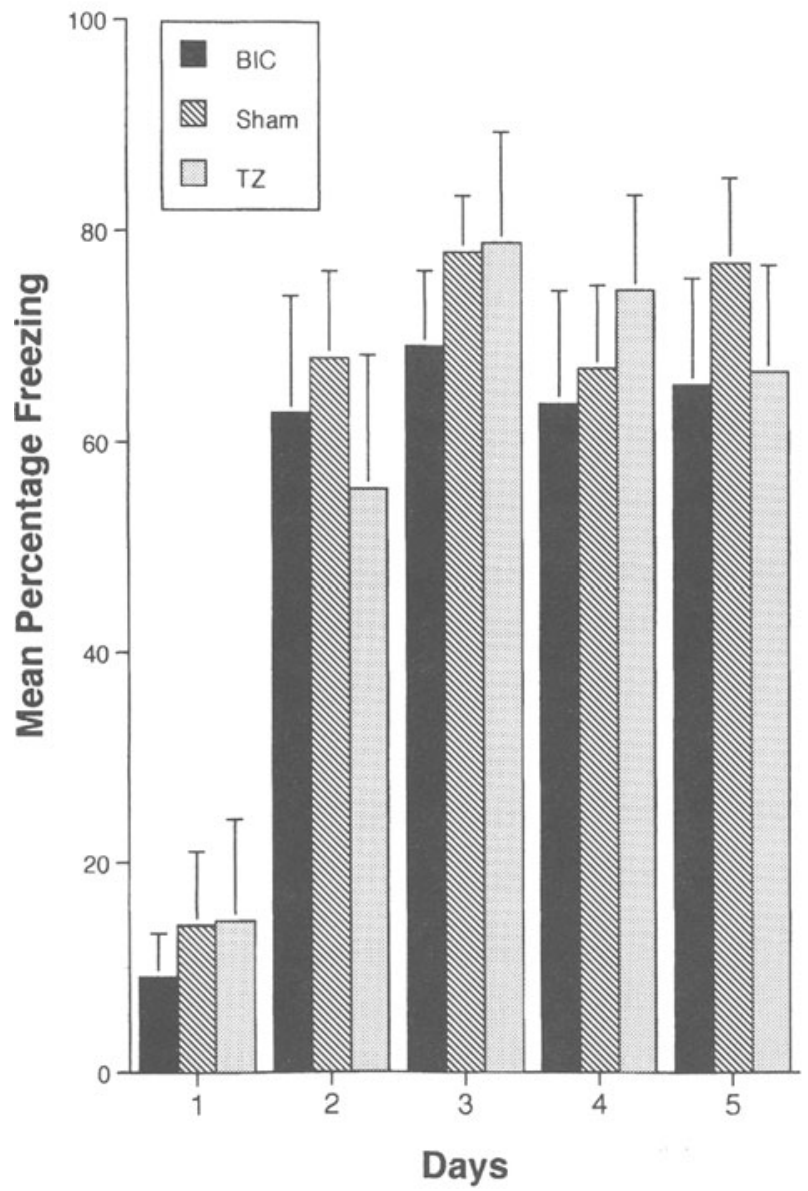

B. Context Change

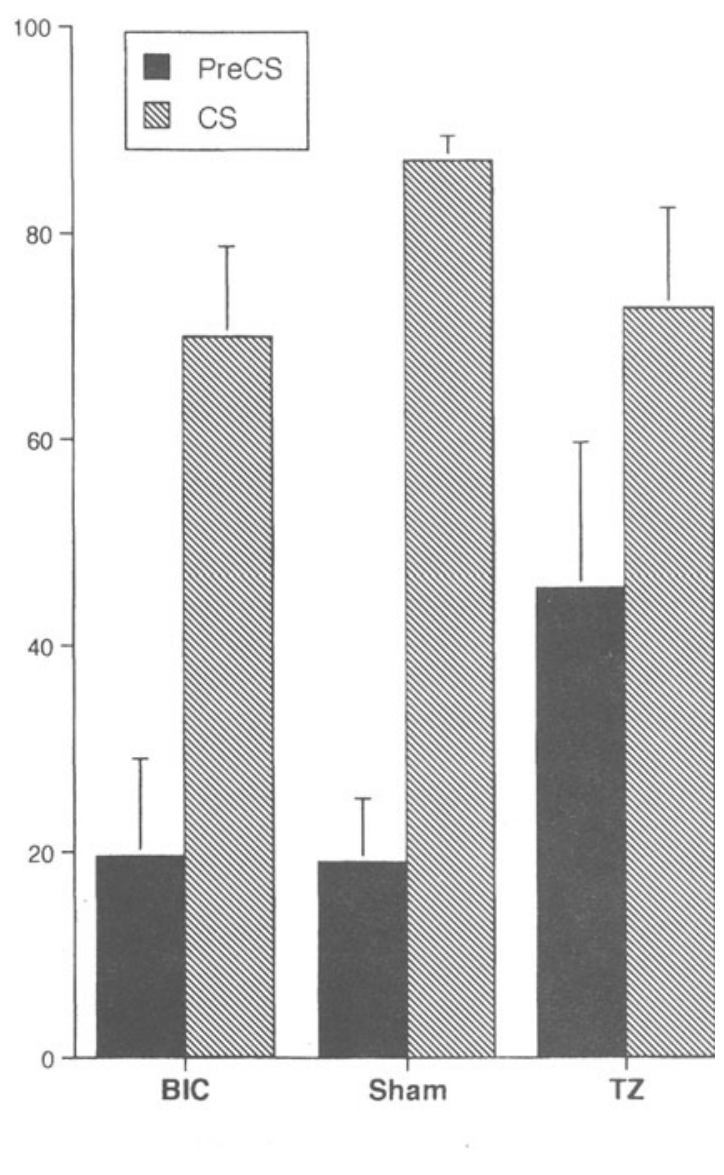

Figure 3. (A) Daily mean percentage freezing ( $+S E$ ) during training to a 20 -sec auditory conditioned stimulus $(\mathrm{CS})$ followed by foot shock over the 5 training days in Experiment 1. The response is shown for the first of the two daily CS trials. (B) Mean percentage freezing $(+S E)$ for the 20 -sec preCS period and for the 20 -sec CS period for the first trial of the 2 test days in the changed context in Experiment 1. No shock was delivered on these test days. The BIC group had lesions to the brachium of the inferior colliculus, the $T Z$ group had lesions to the nucleus of the trapezoid body, and the sham group received sham operations.

the BIC group froze more than the sham group $(p<.05)$ and the TZ group $(p<.001)$, but the difference between the sham and TZ groups did not reach significance $(p=$ .076 ). This overall pattern of differences held over the 5 test days, but the differences among the groups diminished over days and was no longer significant on Day 5 $[F(2,27)=1.82, p>.15]$.

\section{Shock Training}

On the first trial of the first pretraining session, all groups showed a low level of freezing during the preCS period (less than 14\%), and all showed an unconditioned freezing response to the $\mathrm{CS}$. The mean percentage freezing $( \pm S E)$ to the first CS was $46.4 \%( \pm 9.2), 25.6 \%$ $( \pm 10.6)$, and $30.0 \%( \pm 7.2)$ for the BIC, TZ, and sham groups, respectively. An ANOVA showed a significant increase from preCS to $\operatorname{CS}[F(1,27)=22.44, p<.001]$, but neither the group difference $(F<1.0)$ nor the inter- action $[F(2,27)=2.36, p>.10]$ was significant. Response to the CS habituated significantly to the first trial of the second pretraining session $[F(1,27)=24.57, p<.001]$.

The responses to the CS on the first trail of each of the 5 training days are shown in Figure 3A. The data are shown and the analysis is described for only the first of the two daily trials. That trial more clearly showed the conditioning effects without carryover effects from a preceding shock trial. All groups showed an increase in freezing over days, with the most striking increase occurring from Day 1 to Day 2, but there were no consistent group differences. An overall ANOVA showed an effect of days $[F(1,27)=77.81, p<.001]$ but no group difference or interactions $(F \mathrm{~s}<1.0)$. The high degree of freezing to the context largely obscured conditioning to the CS. However, there was a small, but significant, overall difference between the preCS and CS periods $[F(1,27)=5.80, p<$ $.05]$. The mean differences between the preCS and CS pe- 
riods $( \pm S E)$ were $6.9( \pm 4.7), 7.0( \pm 6.2)$, and $10.0( \pm 6.4)$ for the BIC, sham, and TZ groups, respectively.

\section{Context Change}

The context change produced by the change in the test apparatus effectively reduced freezing in the absence of the CS for the BIC and sham groups and less effectively so for the TZ group. On the pretest day in the changed context, the mean percentages freezing $( \pm S E)$ for the blank trials were $7.0 \%( \pm 3.8), 13.2 \%( \pm 6.7)$, and $42.2 \%$ $( \pm 14.8$ ) for the BIC, sham, and TZ groups, respectively. These compare with means of $66.4 \%( \pm 13.2), 61.0 \%$ $( \pm 12.9)$, and $54.4 \%( \pm 16.0)$, respectively, for the first preCS period of the last training session. The decrease in context freezing was significant $[F(1,27)=26.37, p<$ $.001]$, as was the overall group difference for the pretraining session $[F(2,27)=4.28, p<.05]$.

The freezing responses on the first trial of the 2 test days in the changed context are shown in Figure $3 \mathrm{~B}$ collapsed over the 2 test days. The much reduced preCS freezing revealed the extent of freezing to the CS. An ANOVA of these data showed a significant change from preCS to CS $[F(1,27)=104.60, p<.001]$. The group difference was not significant $(F<1.0)$, but the group $X$ period interaction was significant $[F(2,27)=5.88, p<$ $.01]$, reflecting the high level of freezing shown by the TZ group in the preCS period. The interaction was not significant when only the BIC and sham groups were analyzed $[F(1,19)=2.95, p>.10]$. The TZ group, analyzed alone, did show a significant increase in freezing from the preCS period to the CS period $[F(1,8)=6.88, p<.05]$.

\section{Discussion}

The rats with lesions to the BIC showed significantly higher ASR amplitudes than did the sham-operated controls. The BIC-lesioned rats also showed a significantly higher level of freezing induced by the startle stimulus than did the controls. However, they did not differ from the controls in conditioned freezing to a low-intensity auditory CS with a foot-shock US. The rats with lesions to the TZ showed little or no startle response, but their freezing induced by the startle stimulus was only marginally lower than that of the controls. The TZ-lesioned rats did not differ from the controls in acquisition of freezing to a low-intensity auditory CS with a foot-shock US, but the shock experience in this group induced freezing that was more resistant to changes in context than was so for the other two groups.

The BIC lesions did not attenuate freezing to the startle stimulus. On the contrary, the rats with BIC lesions showed significantly higher levels of freezing on the first ASR test day. Although this finding is consistent with our preliminary results (Leaton \& Kelso, 1994), it is surprising in light of the research of LeDoux and his colleagues (e.g., Iwata et al., 1986; LeDoux et al., 1985; LeDoux et al., 1984). They have shown that lesions to the MGN block Pavlovian fear conditioning to an auditory $\mathrm{CS}$, and the BIC provides a major pathway from the auditory brain stem to the MGN. Not only did the BIC lesions fail to attenuate freezing to the auditory stimulus as US in the ASR paradigm, the rats with BIC lesions responded normally to an auditory CS in Pavlovian fear conditioning with a foot-shock US. The auditory specificity of the conditioned response in the BIC-lesioned rats was most clearly shown when the auditory CS was tested in the changed context. In these tests, freezing to the context alone was quite low in the BIC and sham groups but increased dramatically in both groups in the presence of the CS. We must assume that the auditory stimulus as either US or CS can reach the MGN in pathways that run outside of BIC.

The higher than normal level of freezing shown by the BIC group in the ASR paradigm could have several causes: (1) It could be a direct result of that group's increased startle amplitude - that is, a more vigorous startle response might provoke more vigorous freezing, and the more vigorous startle could result from loss of inhibition on either the sensory or the motor side of the reflex. However, the TZ group, which did not show a detectable ASR, did show a substantial level of freezing, making it clear that the ASR and freezing are not directly coupled. (2) The BIC lesions may have reduced tonic inhibition of the acoustic signal as a US input to a fear circuit and thereby increased freezing independent of the ASR. (3) The BIC lesions may have produced a more general loss of inhibition of a fear circuit. The present data do not allow a choice among these options, and the options need not be mutually exclusive.

The increased startle amplitude shown by the BIClesioned rats is consistent with earlier work (Jordan \& Leaton, 1982), which showed increased startle amplitudes with BIC lesions, and with our more recent data (Leaton \& Kelso, 1994) showing enhanced startle amplitudes following lesions to the MGN. These data suggest some form of tonic descending inhibitory influence on the startle circuit that is carried in the BIC. The existence of pathways in the BIC that descend from the auditory cortex to the IC, and perhaps lower brain stem structures, are well known (see, e.g., Harrison \& Howe, 1974; Huffman \& Henson, 1990; Spangler \& Warr, 1991), and recent work (Senatorov \& Hu, 1999) has shown direct projections from MGN to IC. Such descending circuits could serve to inhibit the basic startle circuitry on either its sensory or its motor side. The specific source and target of the inhibitory influence carried in BIC are unknown.

As expected, the rats with lesions to the TZ showed no detectable ASR. Since these lesions invariably severed the tract of the TZ bilaterally, they blocked the auditory input to the startle circuit (Lee, Lopez, Meloni, \& Davis, 1996; Szabó \& Hazafi, 1965). However, the TZ-lesioned rats responded to auditory stimuli in other circumstances. They showed significant unconditioned freezing to an auditory CS, significant acquisition of freezing to the auditory CS with a foot-shock US, and significant freezing to that $\mathrm{CS}$ when tested in a changed context. In these conditions, they differed from the other two groups only 
in the persistence of their context freezing, an unexpected effect for which we can offer no explanation. This high, persistent level of context freezing did not prevent this group from showing significant conditioning to the auditory CS in a changed context (Figure 3B). On balance, the rats with $T Z$ lesions showed a variety of basically normal responses to auditory stimuli even though they could not show an ASR, emphasizing once again that, quite early in its course, the auditory system projects into independent, behaviorally significant circuits (Harrison, 1960).

\section{EXPERIMENT 2}

Experiment 1 has left us with the puzzle: How does the acoustic startle stimulus, as a US for conditioned freezing, gain access to the amygdala? Lesions of BIC should have isolated the MGN from a major source of its input from the IC. The auditory cortex and amygdala, in turn, should have lost significant auditory input from the MGN. Contrary to expectations, the BIC lesions in Experiment 1 increased, rather than reduced, freezing in the ASR paradigm and left conditioning to an auditory CS unaffected. The amygdala, obviously, does not need the auditory information carried in BIC to process an acoustic stimulus as either US or CS for fear conditioning.

The results of Experiment 1 were consistent with our earlier preliminary data (Leaton \& Kelso, 1994) showing that MGN lesions increased freezing and startle amplitude in the ASR paradigm. However, these data appear to conflict with the report of LeDoux et al. (1984) that the acoustic stimulus, as CS, reaches the amygdala via the MGN. Experiment 2 was an attempt to resolve these conflicts. Rats with MGN or BIC lesions were first tested in a standard ASR paradigm followed by a Pavlovian conditioning paradigm with an auditory CS and foot-shock US. They finally were tested for the startle response to a brief foot-shock stimulus. The MGN lesions were significantly enlarged over those in our preliminary report to ensure that the medial portions of the MGN that project directly to the amygdala (LeDoux et al., 1985) were included. The BIC lesions were made electrolytically so as to enlarge them as compared with the BIC knife cuts in Experiment 1 . We wished to avoid any data conflict resulting from inadequately sized lesions.

\section{Method}

\section{Subjects}

Thirty male Long-Evans-derived rats born and raised in the Dartmouth College Psychology Department vivarium were 85-100 days old at the time of surgery. They were maintained on a 14:10-h light:dark cycle (lights on at 0600) and tested between 1000 and $1600 \mathrm{~h}$. They were individually housed and had ad-lib access to food and water. They were divided into three groups matched on body weight (mean weight $=388 \mathrm{~g}$ ). One group (BIC) received bilateral lesions of the brachium of the inferior colliculus $(n=11)$, one group (MGN) received bilateral lesions of the medial geniculate nucleus $(n=11)$, and one group (sham) received sham operations $(n=8)$.

\section{Surgery and Histology}

General surgical and histological procedures were the same as those described for Experiment 1. The bilateral BIC lesions were made electrolytically, rather than with the knife cuts used in Experiment 1 . Two insertions were made on each side $1.3 \mathrm{~mm}$ anterior to the IAL. One insertion was made $2.8 \mathrm{~mm}$ lateral to the midline and $5.5 \mathrm{~mm}$ dorsal to the IAL; the other was made $3.2 \mathrm{~mm}$ lateral and $4.4 \mathrm{~mm}$ dorsal. At each location, the lesion was made with an anodal current of $1.4 \mathrm{~mA}$ for $10 \mathrm{sec}$. The bilateral MGN coordinates were $3.2 \mathrm{~mm}$ anterior and $3.4 \mathrm{~mm}$ dorsal to the IAL and $3.5 \mathrm{~mm}$ lateral to the midline. The anodal current was $2 \mathrm{~mA}$ for $40 \mathrm{sec}$.

\section{Apparatus}

The startle apparatus and the shock-conditioning and contextchange apparatus were the same used in Experiment 1, as were the parameters for the startle stimuli and the $\mathrm{CS}$ in the shock-conditioning paradigm. For testing the startle response to foot shock, the shock stimulus was a $100-\mathrm{msec}, 0.5-\mathrm{mA}$ unscrambled shock delivered through the grid floor of the startle chambers.

\section{Procedure}

Acoustic startle. The general testing procedure was the same as in Experiment 1. Testing began a minimum of 14 days after surgery. Four daily 5-min preexposure sessions in the startle chambers were followed by five eight-trial sessions on consecutive days. Freezing was measured as described for Experiment 1. Observers were blind to group identity.

Shock conditioning. The general shock-training procedure was the same as in Experiment 1. Training began 4 days following the last startle session and continued on consecutive days. One preexposure session in the shock chambers was followed by two daily sessions of CS-alone presentations, followed by 10 daily $C S-$ shock-training sessions. Two trials were presented on a variable 150 -sec intertrial interval on each CS-alone day and training day. For the first 6 training days, the shock was $1 \mathrm{~mA}$ for $0.5 \mathrm{sec}$. For Days 7-10, the shock was increased to $1.6 \mathrm{~mA}$ and $1 \mathrm{sec}$. Freezing was measured during CS presentations as described in Experiment 1 . Seven days after the last training session, the rats were tested in the changed context. On consecutive days, they received (1) four preexposure sessions to the new context with no CS presentations but with freezing measured when the CS would have been presented and (2) four daily sessions with presentation of the CS alone. The CS was presented on the same time schedule as in training, and freezing was measured as before. Observers were blind to group identity.

Shock startle. Five days following the last context-change test, the rats were given one session in the startle chambers with the 100msec, $0.5-\mathrm{mA}$ foot shock as the startle stimulus. They received 20 trials on a $30-\mathrm{sec}$ interstimulus interval. The first stimulus was presented $90 \mathrm{sec}$ into the session, and the rats were removed $60 \mathrm{sec}$ following the last stimulus.

\section{Results}

\section{Histology}

Diagrammatic reconstructions of the largest and smallest MGN lesions are shown on the left in Figure 4. (Histological analysis was done with the experimenter blind to the results of the behavioral tests.) Three rats in the MGN group were excluded from data analysis because their lesions were judged to be incomplete. This usually involved asymmetrical lesions that spared the medial portions of MGN unilaterally. The lesions in the remaining 8 rats extensively damaged all areas of the MGN bilaterally, including the medial suprageniculate nucleus 

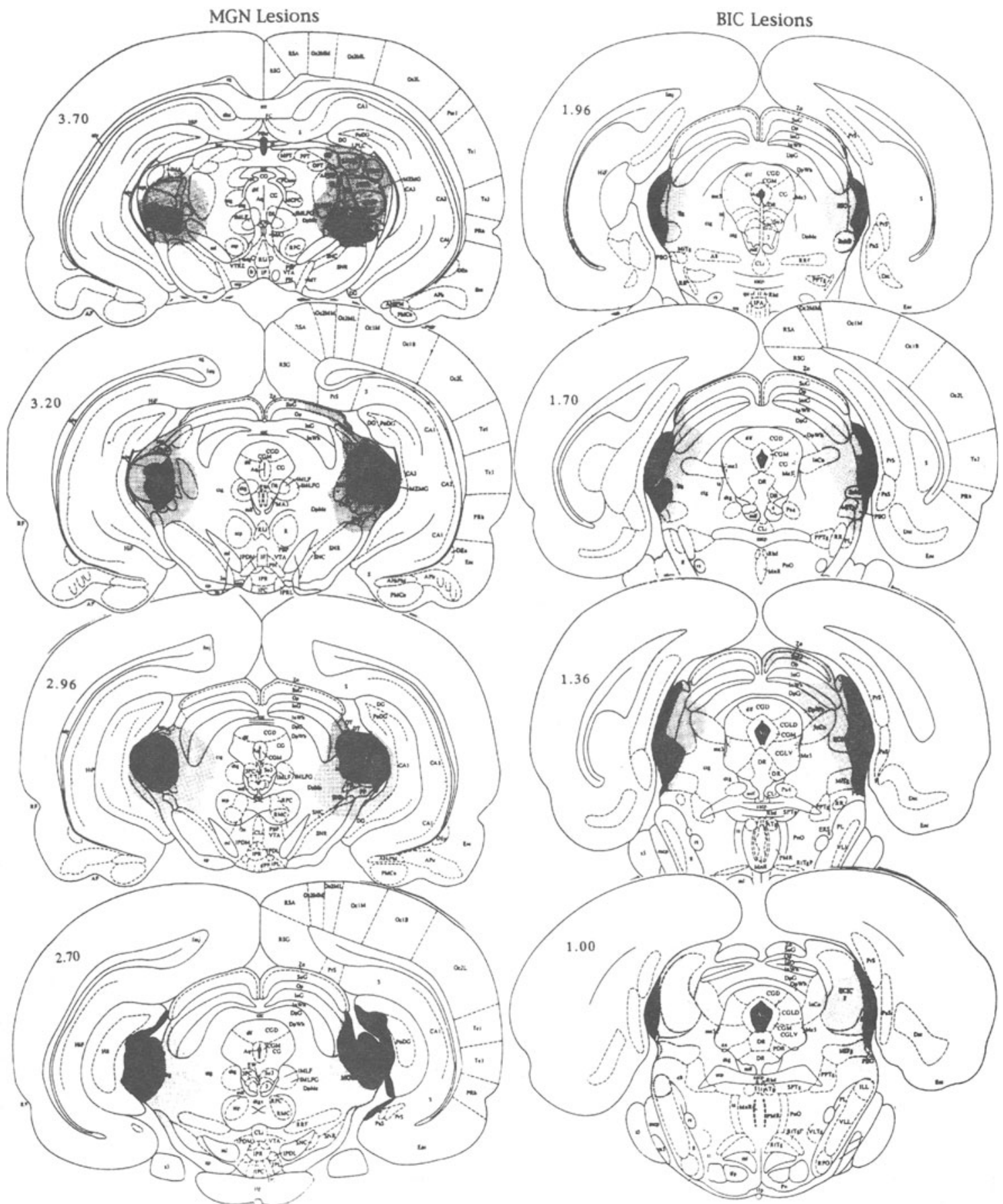

Figure 4. Diagrammatic reconstructions of largest (shaded) and smallest (black) lesions in Experiment 2. The left side of the figure shows lesions to the medial geniculate nucleus (MGN). The right side of the figure shows lesions to the brachium of the inferior colliculus (BIC). The numbers on the left of the diagrams are millimeters anterior to the interaural line. Adapted from The Rat Brain in Stereotaxic Coordinates (2nd ed., Figures 46-48, 50; and 39, 41-43), by G. Paxinos and C. Watson, 1986, New York: Academic Press. Copyright 1986 by Academic Press. Adapted with permission.

and posterior intralaminar nucleus. The larger lesions produced extensive bilateral damage to the anterior pretectal nucleus, damaged the lateral tegmental tract bilaterally, and invaded the central tegmental tract unilaterally. Less extensive and usually unilateral damage occurred to the lateral aspects of the substantia nigra, rostral portions of the BIC, the brachium of the superior colliculus, lateral portions of the superior colliculus, and medial portions of the dentate gyrus.

Diagrammatic reconstructions of the largest and smallest BIC lesions are shown on the right in Figure 4. Three rats in the BIC group were excluded from data 

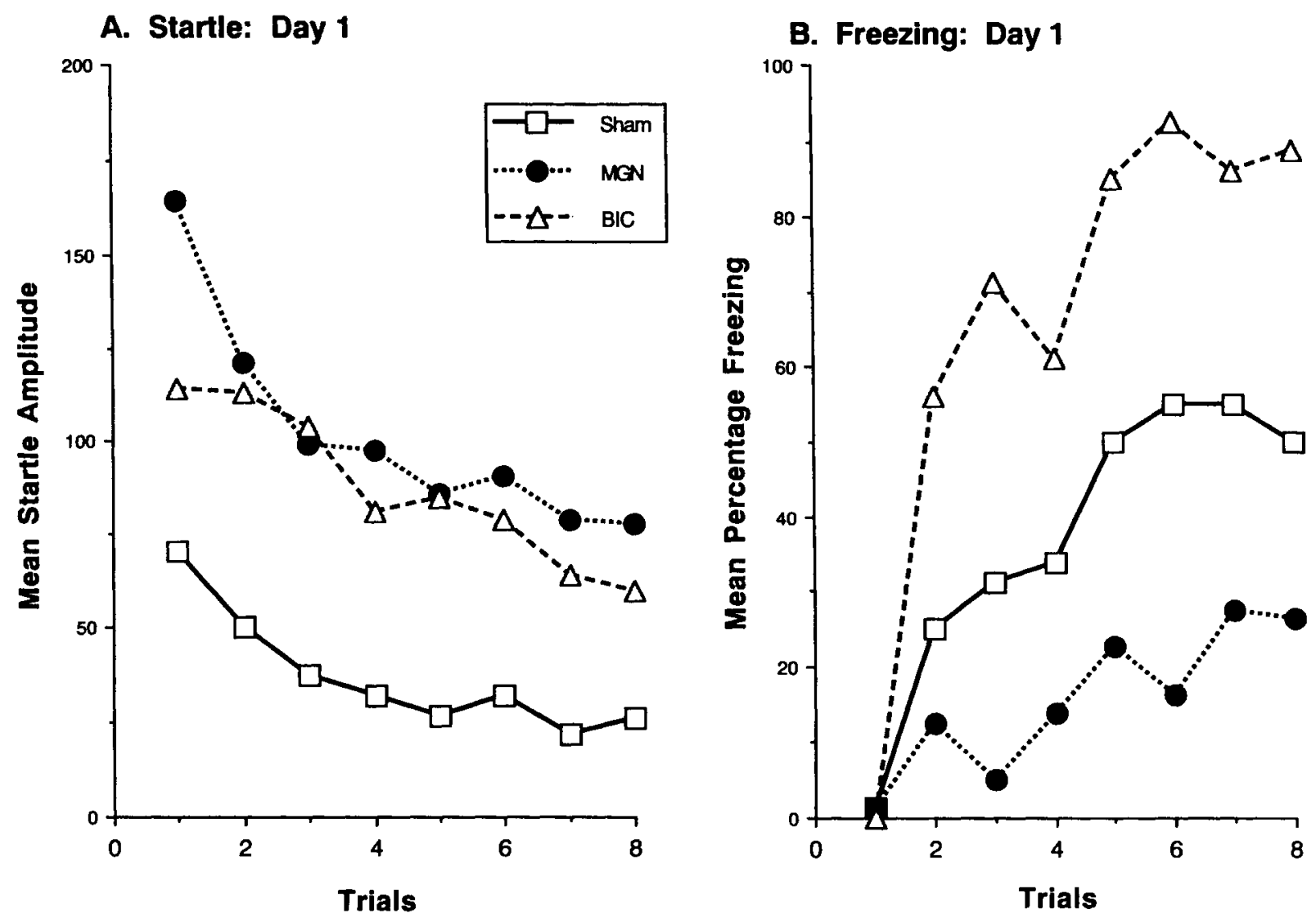

Figure 5. (A) Mean startle amplitude for the three groups over the eight trials on the first acoustic startle response test day in Experiment 2. (B) Mean percentage freezing for the three groups on the first acoustic startle response test day in Experiment 2. Freezing was measured for the 20 sec preceding each of the eight startle stimuli. The BIC group had lesions to the brachium of the inferior colliculus, the MGN group had lesions to the medial geniculate nucleus, and the sham group received sham operations.

analysis because their lesions were judged to be incomplete. This usually involved unilateral or bilateral sparing of the ventral portions of the BIC. The lesions in the remaining 8 rats produced complete loss of BIC throughout its rostral-caudal extent. There was consistent and extensive bilateral damage to the nucleus of BIC and to the parabigeminal nucleus and slight damage to the microcellular tegmental nucleus, the subbrachial nucleus, and the lateral edge of the external cortex of the IC. The larger lesions produced more extensive damage to all of these structures, moderate damage to the intercollicular nucleus, and slight damage to the lateral tegmental tract, the lateral edges of the superior colliculus, and the lateral parts of MGN.

\section{Startle Testing}

Startle. The ASRs for the three groups on the first test day are shown in Figure 5A. The MGN and BIC groups showed similar startle amplitudes that were higher than those of the sham group. An ANOVA over the eight trials yielded a significant group difference $[F(2,21)=6.74$, $p<.01]$ and a significant trial effect $[F(1,21)=33.03$, $p<.001]$. The interaction was not significant $(F<1.0)$ Post hoc analysis (Tukey) of the daily means showed that both the BIC group $(p<.05)$ and the MGN group ( $p<$ .01 ) responded at a significantly higher level than did the sham group, but the BIC and MGN groups did not differ significantly $(p>.70)$. This pattern of differences held over the 5 test days, with the differences between the lesion groups and the sham group diminishing over days. The group difference, while still apparent, was no longer statistically significant by Day $5[F(2,21)=1.13, p>.30]$.

Freezing. The freezing data for the first ASR test day are shown in Figure 5B. The BIC group froze more than the sham group, and the MGN group froze less than the sham group. All groups increased freezing over trials as they experienced the startle stimulus. An ANOVA yielded a significant group difference $[F(2,21)=19.19, p<.001]$, a significant trials effect $[F(1,21)=58.34, p<.001]$, and a significant groups $\times$ trials interaction $[F(2,21)=4.69$, $p<.05]$. Post hoc analysis of the daily means showed that the BIC group froze significantly more than the sham group $(p<.01)$ and the MGN group $(p<.001)$, and the MGN group froze significantly less than the 


\section{A. Shock Training: First Daily CS}

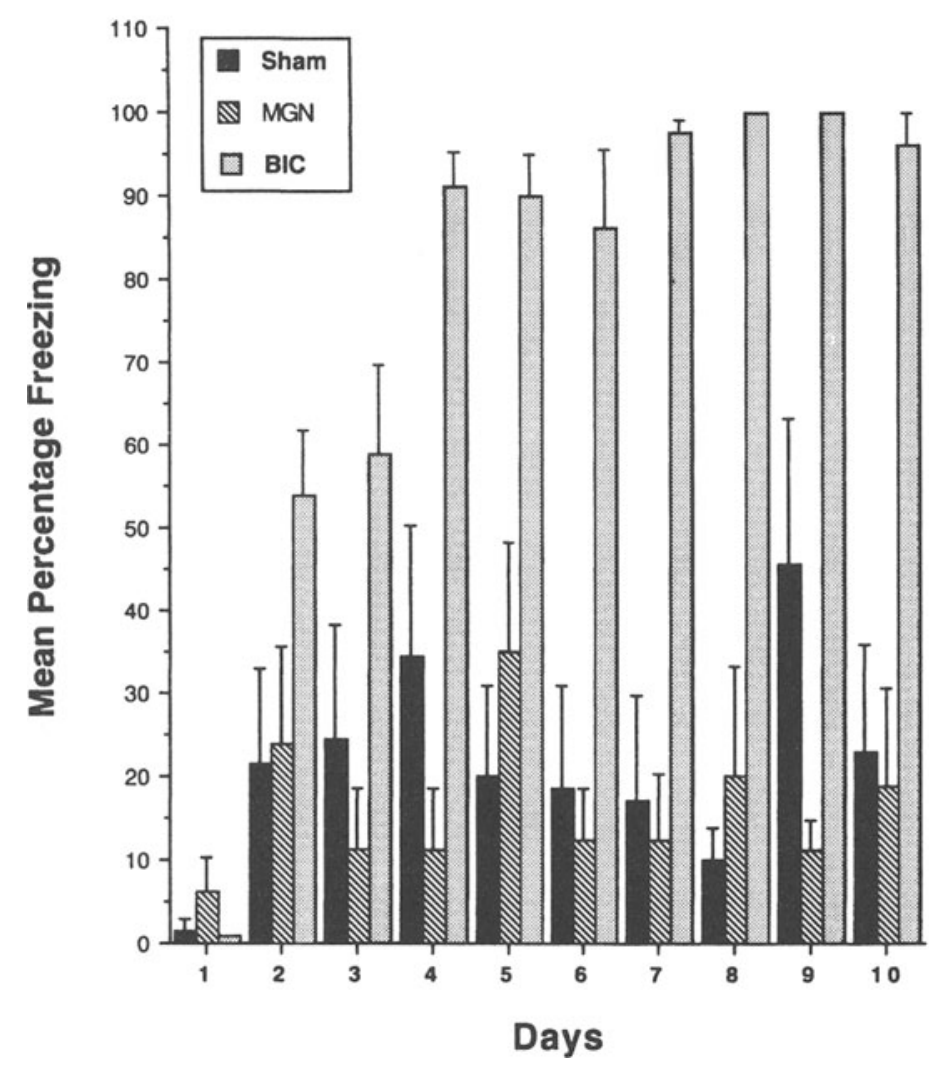

\section{B. Test in Changed Context}

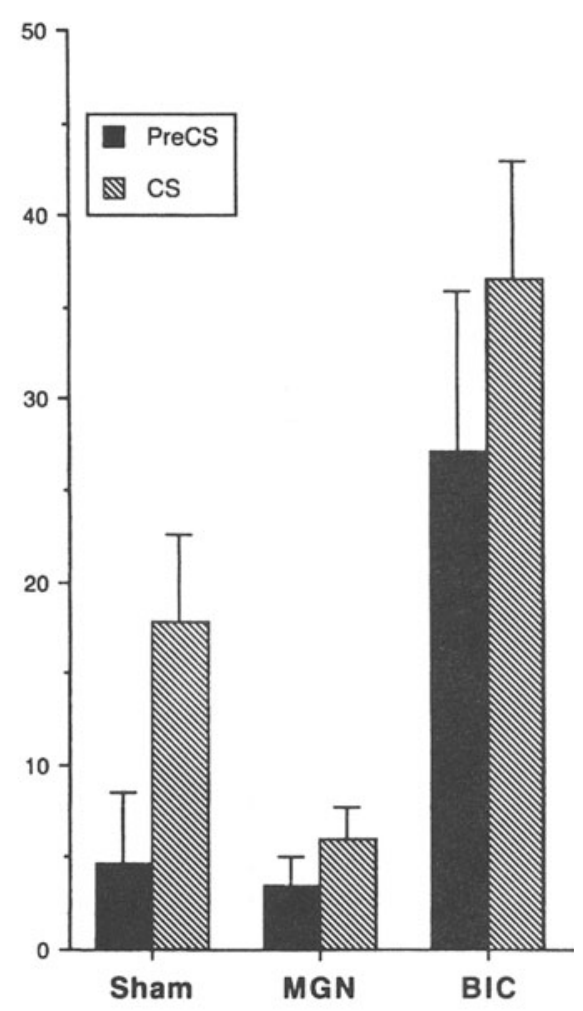

Figure 6. (A) Daily mean percentage freezing $(+S E)$ during training to a 20 -sec auditory conditioned stimulus (CS) followed by foot shock over the 10 training days in Experiment 2 . The response is shown for the first of the two daily CS trials. (B) Mean percentage freezing $(+S E)$ for the 20 -sec preCS period and for the 20-sec CS period for the test days in the changed context in Experiment 2. The data are collapsed over the two trials for each of the 4 test days. No shocks were given during these sessions. The BIC group had lesions to the brachium of the inferior colliculus, the MGN group had lesions to the medial geniculate nucleus, and the sham group received sham operations.

sham group $(p<.05)$. This pattern of differences was maintained over days. After a slight increase in freezing on Day 2 , the overall level of freezing and the differences among the groups decreased over days. The group differences were no longer significant on Day $5(F<1.0)$.

\section{Shock Training}

(One rat in the sham group died before the end of shock training, and its data are not included in any of the shock-session analyses.) During pretraining, there was no freezing by any rats during the preCS period, but all groups showed some unconditioned freezing to the first presentation of the CS. The mean percentages freezing $( \pm S E)$ to the first CS were $4.3( \pm 2.0), 7.5( \pm 4.9)$, and $12.50( \pm 6.2)$ for the sham, MGN, and BIC groups, respectively. An ANOVA showed a significant increase from preCS to $\operatorname{CS}[F(1,20)=7.64, p<.05]$ but no group difference or interaction (both $F \mathrm{~S}<1.0$ ). This unconditioned response to the CS habituated by the second pretraining day. There was no freezing by any rat during ei- ther preCS or CS periods on Day 2. The decrease in responsiveness from Day 1 to Day 2 was significant $[F(1,20)=8.18, p<.05]$.

For shock training, as in Experiment 1, only the first of the two daily trials is shown and analyzed. The percentage freezing for the three groups is shown for the first CS of each of the 10 training days in Figure 6A. All groups increased freezing over days, the BIC group quite dramatically so. An ANOVA over the 10 days showed a significant group difference $[F(2,20)=47.08, p<.001]$, a significant increase over days $[F(1,20)=27.82, p<$ $.001]$, and a significant groups $\times$ days interaction $[F(2,20)=16.25, p<.01]$. Analysis of pairs of groups showed that the sham and MGN groups did not differ significantly $(p>.40)$, but both groups differed significantly from the BIC group (both $p s<.001$ ). The shock level was increased after Day 6 because the sham group showed a much lower level of freezing than did the same group in Experiment 1. There was no apparent effect of this shock-intensity increase, except to perhaps push the 


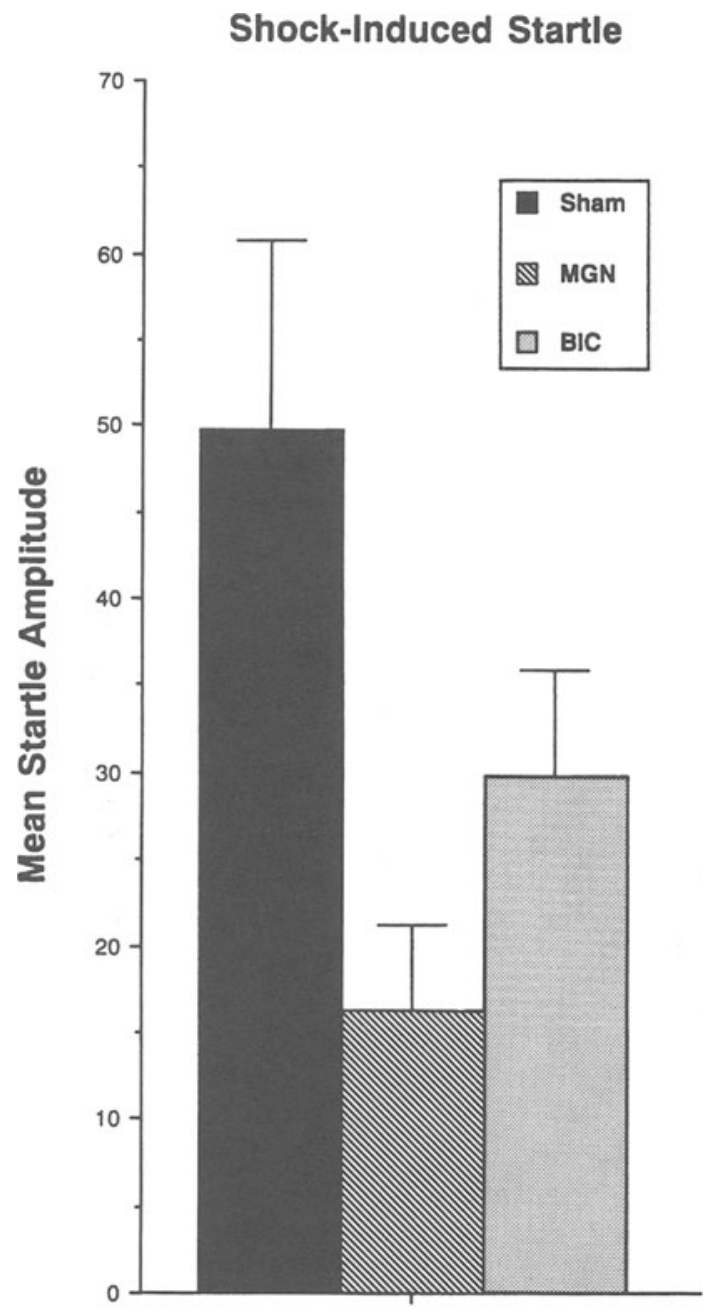

Figure 7. Mean startle amplitude $(+S E)$ to the presentations of a 100-msec, 0.5-mA foot shock for the three groups in Experiment 2. The shocks were presented on a 30-sec interstimulus interval during one 20-trial session. The means shown are for the first 10 trials. The BIC group had lesions to the brachium of the inferior colliculus, the MGN group had lesions to the medial geniculate nucleus, and the sham group received sham operations.

BIC group to a somewhat higher level. Importantly, there was no difference between preCS and CS periods $(F<$ 1.0 ), indicating that this procedure did not differentiate between freezing to the context in the preCS period and freezing to the CS. The same procedure in Experiment 1, for unknown reasons, produced a higher level of freezing in the sham group and a small, but significant, preCS/CS difference in the training context.

\section{Context Change}

The context change effectively reduced freezing in the absence of the CS for all groups, even though the BIC group continued freezing at a relatively high level. The percentages freezing ( $\pm S E$ ) for the first preCS period during the last training session were $37.2 \%( \pm 13.2)$, $25.0 \%( \pm 10.8)$, and $95.0 \%( \pm 3.8)$ for the sham, MGN, and BIC groups, respectively. The mean percentages freezing during the four pretest days in the changed context, measured during the two daily "blank trials," were $1.2 \%( \pm 0.7), 2.2 \%( \pm 1.1)$, and $31.9 \%( \pm 11.2)$ for the sham, MGN, and BIC groups, respectively. The reduction in freezing was significant for all groups $[F(1,20)=$ $42.14, p<.001]$, and the smaller reduction for the BIC group produced a significant interaction $[F(2,20)=3.76$, $p<.05]$. The BIC group, considered separately, did show a significant reduction in freezing to the changed context $[F(1,7)=41.56, p<.001]$. The differences in freezing during the pretest sessions also were significantly different. An ANOVA of the pretest freezing showed a significant group difference $[F(2,20)=6.62, p<.01]$. Post hoc analysis (Tukey) showed that the sham and MGN groups did not differ significantly $(p>.70)$, but both differed significantly from the BIC group (both $p s<.05$ ).

Freezing during testing in the changed context is shown for the preCS and CS periods in Figure 6B. The data are collapsed over the two trials and the 4 test days. An ANOVA for the three groups showed a significant group difference $[F(2,20)=10.78, p<.01]$ and a significant difference between preCS and CS periods $[F(1,20)=$ $13.62, p<.01]$. However, the interaction was not significant $[F(2,20)=1.48, p>.20]$. Comparisons of interest are between the sham group and each of the other two groups. The sham group compared with the MGN group showed a significant group difference $[F(1,13)=5.92$, $p<.05]$ and a significant interaction $[F(1,13)=4.79$, $p<.05]$, showing that the sham group increased more to the CS than did the MGN group. The sham group compared with the BIC group showed a significant group difference $[F(1,13)=5.99, p<.05]$, but the interaction did not approach significance $(F<1.0)$. The elevated level of preCS freezing shown by the BIC group did not prevent that group from showing an increase in freezing to the CS that was comparable to the controls.

\section{Shock-Induced Startle}

The mean startle amplitudes for the first 10 trials for the shock-induced startle test for the three groups are shown in Figure 7. Only the first 10 trials were analyzed because the addition of the final ten trials served simply to dilute the group differences. The sham group showed higher amplitudes than did the two lesioned groups, and the BIC group showed somewhat higher amplitudes than did the MGN group. A one-way ANOVA of the mean for these 10 trials yielded a significant group difference $[F(2,20)=4.97, p<.05]$. Post hoc analysis showed that the sham group responded at a significantly higher level than the MGN group $(p<.05)$, but the sham and BIC groups $(p>.15)$ and the MGN and BIC groups $(p>.60)$ did not differ significantly.

\section{GENERAL DISCUSSION}

Compared with sham-operated controls, the rats with MGN lesions in Experiment 2 showed higher startle amplitude but reduced levels of freezing during ASR test- 
ing. The rats with BIC lesions showed both higher startle amplitude and higher levels of freezing than did the controls during ASR testing, consistent with the results of Experiment 1 . In a subsequent test of startle induced by a brief, low-intensity foot shock, the MGN and BIC groups showed lower startle amplitudes than did the controls, the MGN group significantly so. In Pavlovian fear conditioning with an auditory CS and foot-shock US, MGN lesions significantly attenuated freezing to the CS when context conditioning was controlled. The rats with BIC lesions, on the other hand, responded normally to the $\mathrm{CS}$ in fear conditioning in Experiments 1 and 2, although this group showed a heightened level of freezing throughout fear conditioning in Experiment 2. The TZ lesions in Experiment 1 abolished the ASR yet left the rats capable of showing fear to an acoustic US or CS and making an unconditioned response to an acoustic CS.

The results for the MGN lesions provide at least a partial answer to the question posed in the introduction: How does the acoustic startle stimulus, as a US for conditioned freezing, gain access to the amygdala? It must pass through the MGN. We know that lesions to the amygdala block the freezing response in the ASR paradigm (Young \& Leaton, 1996), as did the lesions to MGN in Experiment 1. These data parallel the results of Bellgowan and Helmstetter (1996), who showed that lesions of the MGN, as well as lesions of the amygdala, blocked the unconditioned hypoalgesia induced by an acute, prolonged white-noise stimulus. The present data are consistent with the work of LeDoux and others (Campeau \& Davis, 1995; Iwata et al., 1986; LeDoux et al., 1984) showing that an acoustic stimulus as CS in a fearconditioning paradigm reaches the amygdala through the MGN. The MGN lesions in Experiment 1 were similar in effect to these previous reports in that they attenuated fear conditioning to an acoustic CS. Apparently, an acoustic stimulus as US reaches the amygdala through the same basic neural pathways as does an acoustic stimulus as CS.

The present MGN data are not consistent with our preliminary report (Leaton \& Kelso, 1994) in which MGN lesions did not reduce (but in fact increased) freezing in the ASR paradigm. Those earlier MGN lesions were smaller than those reported here, and, perhaps more importantly, they were more laterally placed and spared the more medial portions of the MGN. Direct projections from MGN to the amygdala originate in the medial division of MGN, including the posterior intralaminar nucleus and the medial posterior nucleus (LeDoux, Farb, \& Ruggiero, 1990; LeDoux et al., 1985), and these projections are sufficient for fear conditioning to an acoustic CS (Romanski \& LeDoux, 1992). Perhaps these direct projections to the amygdala are also sufficient for fear conditioning with an acoustic stimulus as US.

The data from the BIC lesions in Experiments 1 and 2 indirectly suggest the importance of the medial division of the MGN for the freezing response. Rather than reducing freezing induced by the acoustic startle stimulus, BIC lesions actually increased freezing in the ASR paradigm. Therefore, the fear-inducing dimensions of the startle stimulus must reach the MGN -and, thus, the amygdalain pathways that run outside of BIC. BIC is the primary pathway between IC and MGN, and it carries projections largely to the ventral and dorsal divisions of MGN (Helfert, Snead, \& Altschuler, 1991; Winer, 1991). In addition to projections from IC that may run in BIC, the medial division of MGN receives rich projections from pathways that run ventral and medial to BIC. One such pathway is the lateral tegmental system, which has origins in the nuclei of the lateral lemniscus and other brain stem structures in cats (Morest, 1965; Winer, 1991). LeDoux and his colleagues (LeDoux et al., 1990; LeDoux et al., 1985) have shown direct projections from the nuclei of the lateral lemniscus to the medial division of the MGN in rats, and these projections may contribute to a medial pathway. Our BIC lesions left intact these medial pathways, and animals with these lesions could respond to an acoustic stimulus as either US or CS for fear conditioning.

Although the MGN and BIC lesions had opposite effects on freezing in the ASR paradigm, both lesion types increased startle amplitudes, suggesting a descending tonic inhibition on the ASR circuitry. This descending influence appears to travel in BIC and course through or originate in MGN. Efferent fibers from the auditory cortex terminate primarily in MGN and IC but may project to lower brain stem structures as well. IC also receives descending projections from somatosensory cortex. IC in turn projects widely to lower auditory structures. (For a review of these descending pathways, see Harrison \& Howe, 1974, Huffman \& Henson, 1990, and Spangler \& Warr, 1991.) Descending pathways originating in MGN have been considered sparse or nonexistent (Adams, 1980; Andersen, Snyder \& Merzenich, 1980), but Senatorov and $\mathrm{Hu}(1999)$ have recently shown a direct pathway from MGN to IC in rats. Thus, the known anatomy suggests that the source of the descending inhibition could be either the auditory cortex or the MGN itself. The immediate target for this inhibition could be the IC or lower brain stem structures.

If the auditory cortex is the source of the descending inhibition, then one would expect to find unambiguous increases in the amplitude of the ASR following cortical damage. Unfortunately, the little available data do not support this view. Groves, Wilson, and Boyle (1974) reported a reduction in startle amplitude following auditory cortex lesions in rats. In mice, Hunter and Willott (1993) reported a small increase in startle amplitude 1 day after auditory cortex ablation but no effect 1 month later. Severing the pathways between auditory cortex and the brain stem by decerebration has produced ambiguous results on ASR amplitude in rats. Szabó and Hazafi (1965) and Davis and Gendelman (1977) reported no change in startle amplitude after acute decerebrations, whereas Leaton, Cassella, and Borszcz (1985) reported increased ASR amplitude in chronic decerebrates. However, many factors may confound ASR amplitude comparisons between 
decerebrates and intact controls. Acute decerebration may produce spinal shock, some chronic decerebrates never recover adequately for testing, motor and postural changes may confound response measures in both acute and chronic preparations, and it is difficult to maintain comparable body weights in chronic decerebrates and controls. Other than the present data and our previous reports, we know of no reports of ASR amplitude changes following MGN damage that would suggest the MGN as a source of the descending inhibition.

IC is a target for many of the projections from the auditory cortex and MGN, and it in turn projects throughout the lower brain stem. Thus, IC is a likely area to modulate the ASR in response to descending influences. Unfortunately, the data from damage to IC is inconsistent. Leitner and Cohen (1985) found dramatic increases in startle amplitude following large bilateral radio frequency lesions to IC in rats. This is just the kind of outcome to be expected if a descending influence inhibits an excitatory drive on ASR from the IC. However, more limited, but still large IC lesions in rats abolished the ASR acutely, but response amplitude fully recovered to normal levels (Groves et al., 1974). Jordan and Leaton (1983) found that rather large IC lesions produced either small attenuating or small potentiating effects, depending on the specifics of testing. In mice, Parham and Willott (1990) found that small increases in ASR amplitude resulted only from lesions to specific nuclear subdivisions of IC.

These data leave us uncertain about both the source and the target of the proposed tonic inhibitory effect on ASR. Damage to the auditory cortex does not produce the consistent changes in ASR amplitude to be expected if it were the source of the inhibition. Only the present data, using axon-damaging electrolytic lesions, implicate the MGN as the source of the inhibition. Only the Leitner and Cohen (1985) data are consistent with the IC as a target for the inhibition, and their lesions were too large to allow specific conclusions about the IC. We know very little of potentiating effects on ASR amplitude of structures in the lower brain stem that could be direct targets of fibers carried in BIC. Lesions to the pedunculopontine tegmental nucleus, for example, can significantly increase ASR amplitude (Swerdlow \& Geyer, 1993), and this nucleus plays a critical role in the modulation of ASR by prepulse inhibition (Koch, 1999). However, no evidence shows this nucleus a target of BIC fibers. A very speculative circuit would have the MGN as the source of the inhibition with an immediate target near enough to the IC to have been damaged by the Leitner and Cohen lesions, but research has barely begun to identify this circuitry.

In the shock-induced startle tests, the rats with BIC or MGN lesions showed lower startle amplitudes than did the controls. This result indicates that BIC or MGN lesions do not simply produce generally hyperreactive animals and suggests that the effect of the lesions on startle amplitude is at least partially specific to the ASR. If the effect is specific to the ASR, then the results further suggest that the descending inhibition carried in BIC has its influence on the sensory side of the ASR circuitry. We assume, consistent with the data of Leitner, Powers, and Hoffman (1980), that the ASR and the shock-induced startle use the same motor pathway. This result must be qualified since the shock-induced startle test was completed late in the sequence of testing, whereas the ASR tests began the series. However, it was the only test in which the direction of the startle amplitude difference between the lesioned groups and controls was reversed.

The BIC-lesioned group in Experiment 2 replicated the results of the same group in Experiment 1 in all of the ASR tests. Both groups also showed conditioned freezing to the $\mathrm{CS}$ in the Pavlovian conditioning training and testing sessions. However, the BIC group in Experiment 2 showed significantly more freezing than either the sham or the MGN group in the Pavlovian conditioning sessions and maintained a relatively high level of contextual, preCS freezing in the changed context. The BIC group in Experiment 1 did not differ from controls during these sessions. As can be seen by comparing Figures 1 and 4, the BIC lesions in Experiment 1 (knife cuts) were rather well restricted to the lateral edge of the brain stem, whereas the BIC lesions in Experiment 2 (electrolytic) invaded more medial structures and extended more posteriorly. The change in lesion type in Experiment 2 was intentionally used to produce larger lesions so as to guarantee that we interrupted all of the lateral pathways. The cost was to produce more damage outside of BIC, which apparently produced the unexpected changes in responsiveness in the foot-shock paradigm. It is impossible to know from these experiments whether damage to specific structures outside of BIC created the enhanced freezing in the Pavlovian conditioning phase of Experiment 2, but it seems reasonable to assume that the common effects in the ASR paradigm were produced by the common damage to the lateral aspects of the brain stem, BIC. It should be noted again that the larger lesions in the BIC group in Experiment 2 did not render those rats generally hyperreactive. They were less responsive than controls when startle was induced by a low-intensity foot shock.

These data have produced an unexpected dissociation of the behavioral effects of damage to two primary structures in the auditory pathways. Lesions to BIC or MGN increased ASR amplitude, whereas BIC lesions increased freezing to the acoustic startle stimulus and MGN lesions reduced freezing to this same stimulus. These data can best be explained by hypothesizing two separate neural pathways. One is a descending inhibitory pathway that travels through MGN and BIC to reach IC and lower auditory structures. This pathway provides tonic inhibition on the sensory side of the ASR circuit. The second is an ascending pathway that runs medial to BIC and carries the fear-inducing dimensions of the acoustic stimulus to the amygdala by way of MGN. The source of this pathway is unknown, but it is clear that the fear-inducing dimensions of the acoustic stimulus, whether as CS or US, are separated from other aspects of the stimulus below the level of MGN and perhaps below the level of IC. Both the 
descending pathway and the ascending pathway serve to adjust the amplitude of the acoustic startle response: the descending pathway by direct inhibition of the startle circuit, and the ascending pathway by influencing fear that can indirectly affect startle amplitude.

\section{REFERENCES}

ADAMS, J. C. (1980). Crossed and descending projections to the inferior colliculus. Neuroscience Letters, 19, 1-5.

Andersen, R. A., Snyder, R. L., \& Merzenich M. M. (1980). The topographic organization of corticocollicular projections from physiologically identified loci of AI, AII, and anterior cortical fields of the cat. Journal of Comparative Neurology, 191, 479-494.

Bellgowan, P. S. F., \& Helmstetter, F. J. (1996). Neural systems for the expression of hypoalgesia during nonassociative fear. Behavioral Neuroscience, 110, 727-736.

Borszcz, G. S., Cranney, J., \& Leaton, R. N. (1989). Influence of long-term sensitization on long-term habituation of the acoustic startle response in rats: Central gray lesions, preexposure, and extinction. Journal of Experimental Psychologv: Animal Behavior Processes, 15, 54-64.

CAmpeau, S., \& Davis, M. (1995). Involvement of subcortical and cortical afferents to the lateral nucleus of the amygdala in fear conditioning measured with fear-potentiated startle in rats trained concurrently with auditory and visual stimuli. Journal of Neuroscience, 15, $2312-2327$.

Davis. M. (1992). The role of the amygdala in fear and anxiety. Annual Review of Neuroscience, 15, 353-375.

Davis, M.. \& Gendelman. P. M. (1977). Plasticity of the acoustic startle response in the acutely decerebrate rat. Journal of Comparative \& Physiological Psychology, 91, 549-563.

FANSELOW, M. (1984). Shock-induced analgesia on the formalin test: Effects of shock severity, naloxone, hypophysectomy, and associative variables. Behavioral Neuroscience, 98, 79-95.

Grossman, S. P., Grossman. L., \& Walsh. L. (1975). Functional organization of the rat amygdala with respect to avoidance behavior. Journal of Comparative \& Phvsiological Psychologv, 88, 829-850.

Groves, P. M., Wilson, C. J., \& Boyle, R. D. (1974). Brain stem pathways, cortical modulation, and habituation of the acoustic startle response. Behavioral Biologv, 10, 391-418.

HARRISON, J. M. (1960). Some behavioral functions of the lower auditory pathways in the rat. Journal of Comparative Neurology. 115. 143-154

HARRISON. J. M.. \& HowE, M. E. (1974). Anatomy of the descending auditory system (mammalian). In W. D. Keidel \& W. D. Neff (Eds.), Handbook of sensory phvsiologv (Vol. V, Part 1, pp. 363-388). Berlin: Springer-Verlag.

Helfert, R. H.. Snead. C. R.. \& Altschuler. R. A. (1991). The ascending auditory pathways. In R. A. Altschuler, R. P. Bobbin, B. M. Clopton, \& D. W. Hoffman (Eds.), Neurobiology of hearing: The central auditory svstem (pp. 1-25). New York: Raven.

Helmstetter. F. J. (1992). Contribution of the amygdala to learning and performance of conditioned fear. Physiology \& Behavior, 51, $1271-1276$.

HitchсосK, J., \& Davis, M. (1986). Lesions of the amygdala, but not of the cerebellum or red nucleus, block conditioned fear as measured with the potentiated startle paradigm. Behavioral Neuroscience, 100 , 11-22.

HufFMAN, R. F., \& HeNSON, O. W., JR. (1990). The descending auditory pathway and acousticomotor systems: Connections with the inferior colliculus. Brain Research Reviews, 15, 295-323.

Hunter, K. P., \& WillotT, J. F. (1993). Effects of bilateral lesions of auditory cortex in mice on the acoustic startle response. Physiology \& Behavior, 54, 1133.1139.

iwata, J.. LeDoux, J. E., Meeley, M. P., Arneric, S.. \& Reis. D. J. (1986). Intrinsic neurons in the amygdaloid field projected to by the medial geniculate body mediate emotional responses conditioned to acoustic stimuli. Brain Research, 383, 195-214.

JORDAN, W. P., \& LEATON, R. N. (1982). Startle habituation in rats after lesions in the brachium of the inferior colliculus. Physiology \& Behavior, 28, 253-258.

JORDAN W. P., \& LEATON R. N. (1983). Habituation of the acoustic startle response in rats after lesions in the mesencephalic reticular formation or in the inferior colliculus. Behavioral Neuroscience, 97, 710-724.

Kapp, B. S., Frysinger, R. C., Gallagher, M., \& Haselton, J. R. (1979). Amygdala central nucleus lesions: Effect on heart rate conditioning in rabbits. Physiology \& Behavior, 23, 1109-1117.

Косн, M. (1999). The neurobiology of startle. Progress in Neurobiology, 59, 107-128.

LEATON, R. N. (1976). Long-term retention of the habituation of lick suppression and startle response produced by a single auditory stimulus. Journal of Experimental Psychology: Animal Behavior Processes, 2, 248-259.

Leaton, R. N., Cassella, J. V., \& Borszcz, G. S. (1985). Short-term and long-term habituation of the acoustic startle response in chronic decerebrate rats. Behavioral Neuroscience, 99, 901-912.

Leaton, R. N., \& CRANNEY, J. (1990). Potentiation of the acoustic starthe response by a CS paired with an acoustic startle stimulus in rats. Journal of Experimental Psychology: Animal Behavior Processes, 16, 279-287.

Leatron, R. N., \& Kelso, J. M. (1994). Medial geniculate lesions: Startle amplitude, habituation, and freezing in an acoustic startle paradigm. Society for Neuroscience Abstracts, $20,799$.

LEATON, R. N., \& SupPl.E. W. F., JR. (1987). The amygdala and longterm habituation of the acoustic startle response and freezing in rats. Society for Neuroscience Abstracts, 13, 644.

LEDoux, J. E. (1995). Emotion: Clues from the brain. Annual Review of Psychology, 46, 209-235.

LeDoux, J. E., FARB, C., \& Ruggiero, D. A. (1990). Topographic organization of neurons in the acoustic thalamus that project to the amygdala. Journal of Neuroscience, 10, 1043-1054.

LeDoux, J. E., Ruggiero, D. A., \& Reis, D. J. (1985). Projections to the subcortical forebrain from anatomically defined regions of the medial geniculate body in the rat. Journal of Comparative Neurolog. $, 242,182-213$.

LeDoux, J. E., Sakaguchi, A., \& Reis, D. J. (1984). Subcortical efferent projections of the medial geniculate nucleus mediate emotional responses to conditioned acoustic stimuli. Journal of Neuroscience, 4, 683-698.

Lee, Y., Lopez, D. E., Meloni, E. G., \& Davis, M. (1996). A primary acoustic startle pathway: Obligatory role of cochlear root neurons and the nucleus reticularis pontis caudalis. Journal of Neuroscience, 16, 3775-3789.

LEITNER, D. S., \& COHEN, M. E. (1985). Role of the inferior colliculus in the inhibition of acoustic startle in the rat. Physiology \& Behavior. 34, 65-70.

Leitner, D. S., Powers, A. S., \& Hoffman. H. S. (1980). The neural substrate of the startle response. Physiology \& Behavior, 25, 291-297.

MOREST, D. K. (1965). The lateral tegmental system of the midbrain and medial geniculate body: A study with Golgi and Nauta methods in the cat. Journal of Anatomy, 99, 611-634.

Parham, K., \& Willott, J. F. (1990). Effects of inferior colliculus lesions on the acoustic startle response. Behavioral Neuroscience, 104 . $831-840$

Paxinos, G., \& Watson, C. (1986). The rat brain in stereotaxic coordinates (2nd ed.). New York: Academic Press.

Phillips, R. G., \& LeDoux, J. E. (1992). Differential contribution of amygdala and hippocampus to cued and contextual fear conditioning. Behavioral Neuroscience, 106, 274-285.

RomANSKI, L. M.. \& LEDouX, J. E. (1992). Equipotentiality of thalamoamygdala and thalamo-cortico-amygdala circuits in auditory fear conditioning. Journal of Neuroscience, 12, 4501-4509.

Senatorov, V. V., \& Hu. B. (1999). System of the descending projections from the auditory thalamus to the inferior colliculus in the rat. Society for Neuroscience Abstracts, 25, 1418.

SPANGLER, K. M., \& WARR, W. B. (1991). The descending auditory system. In R. A. Altschuler, R. P. Bobbin, B. M. Clopton, \& D. W. Hoffman (Eds.), Neurobiology of hearing: The central auditory system (pp. 27-45). New York: Raven. 
Swerdlow, N. R., \& Geyer, M. A. (1993). Prepulse inhibition of acoustic startle in rats after lesions of the pedunculopontine tegmental nucleus. Behavioral Neuroscience, 107, 104-117.

SzaBó, l., \& HAZAFI, K. (1965). Elicitability of the acoustic startle reaction after brain stem lesions. Acta Physiologica Academiae Scientiarum Hungarica, 27, 155-165.

WINER, J. A. (1991). Anatomy of the medial geniculate body. In R. A. Altschuler, R. P. Bobbin, B. M. Clopton, \& D. W. Hoffman (Eds.), Neurobiology of hearing: The central auditory system (pp. 293-333). New York: Raven.

Young, B. J., Helmstetter, F. J., Rabchenuk, S. A., \& Leaton, R. N. (1991). Effects of systemic and intra-amygdaloid diazepam on long- term habituation of acoustic startle in rats. Pharmacology, Biochemistry \& Behavior, 39, 903-909.

Young, B. J., \& Leaton, R. N. (1994). Fear potentiation of acoustic startle stimulus-evoked heart-rate changes in rats. Behavioral Neuroscience, 108, 1065-1079.

Young, B. J., \& LeATON, R. N. (1996). Amygdala central nucleus lesions attenuate acoustic startle stimulus-evoked heart-rate changes in rats. Behavioral Neuroscience, 110, 228-237.

(Manuscript received December 16, 1999; revision accepted for publication May 8, 2000.) 